\title{
MANTEGAZZA E GLI STUDENTI UNIVERSITARI NEGLI ANNI '89-'95
}

\author{
S.E. MONS. FRANCESCO GIOVANNI BRUGNARO (*)
}

SunTo. - Con il suo intervento, Mons. Francesco Giovanni Brugnaro, Arcivescovo di Camerino - San Severino Marche, offre una personale testimonianza della sua esperienza di sacerdote, di amico e di collega del Magnifico Rettore Prof. Paolo Mantegazza, incontrato negli anni '89-'95 durante il suo servizio di Cappellano presso la Rettoria Santa Maria Annunciata dell'Università degli Studi di Milano. Al ricordo affettuoso e grato verso l'esimia figura di rettore, di padre e di maestro, si aggiungono alcune caratteristiche riguardanti il rapporto che il Prof. Mantegazza sapeva intessere con gli studenti universitari, attento a considerare la persona nel suo insieme piuttosto che come soggetto impersonale ed anonimo. Questa speciale attenzione scaturiva dalla profonda convinzione che l'educazione è molto più del semplice insegnamento. Egli introdusse nella sua lunga esperienza di docente quel di più che trasformava i suoi corsi in vere e proprie scuole di vita: l'apporto umano ed etico per affrontare le sfide future più difficili nell'ambito professionale. Per questo egli fu anche grande mediatore presso i valentissimi docenti affinché mantenessero alto il rapporto tra ricerca scientifica e didattica, tra qualità della preparazione al dottorato-specializzazione e serietà morale e metodologica del futuro professionista. Nella memoria di Mons. Brugnaro è conservato il dolore che colpì la famiglia Mantegazza per la tragica e prematura scomparsa dei due amatissimi figli. Ispirato anche dal carisma di Don Giussani, insieme alla moglie Andreina, egli seppe trasformare quel dolore lacerante in testimonianza esemplare di vita cristiana fondata sulla Risurrezione di Cristo. La comune frequentazione dell'Arcivescovo di Milano dal 1979 al 2002, ha permesso ad entrambi di vivere una fede quotidiana, attinta alla visione profetica della pastorale biblica del Card. Martini anche all'interno dell'Università, stimolando la cattedra dei non credenti.

$* * *$

ABSTRACT. - With his report, Mons. Francesco Giovanni Brugnaro, Archbishop of Camerino - San Severino Marche, offers a personal testimony of his experience as a priest, friend and colleague of the Rectoral Prof. Paolo Mantegazza, who he met in the years '89-'95 during his chaplain service at the Rectory of Santa Maria Annunciata of

(*) Arcivescovo di Camerino - San Severino Marche, Italia.

E-mail: segreteria.arcivescovo@arcidiocesicamerino.it 
the University of Milan. In addition to the affectionate and grateful remembrance towards the exemplary role of rector, father and teacher, he adds some characteristics regarding the relationship that Prof. Mantegazza knew to weave with the university students, careful to consider the person as a whole rather than as a subject impersonal and anonymous. This special attention stemmed from the profound conviction that education is much more than simple teaching. He introduced in his long experience as a teacher the one that transformed his courses into real schools of life: the human and ethical contribution to face the most difficult future challenges in the professional field. For this reason he was also a great mediator for the very talented teachers to keep the relationship between scientific and didactic research high, between the quality of the preparation for the doctorate-specialization and the moral and methodological seriousness of the future professional. In the memory of Mons. Brugnaro, the pain that struck the Mantegazza family for the tragic and premature disappearance of the two beloved sons is preserved. Inspired also by Don Giussani's charism, together with his wife Andreina, he was able to transform that lacerating pain into an exemplary witness of Christian life founded on the Resurrection of Christ. The common attendance of the Archbishop of Milan from 1979 to 2002, allowed both to live a daily faith, drawn from the prophetic vision of the biblical pastoral of Card. Martini also within the University, stimulating the chair of non-believers.

1. Raggiunto nei mesi scorsi dalla notizia della scomparsa del Prof. Paolo Mantegazza, un'inattesa ondata di ricordi ha fatto capolino in me, insieme alla necessità di condividerli e di fissarli in modo duraturo a motivo dell'altezza e della significativa credibilità di questa bellissima figura di uomo di governo accademico, di medico e di maestro.

Conservo viva nella memoria il dono di averlo avuto come Rettore dell'Università degli Studi (detta Statale) di Milano negli anni in cui svolgevo il mio ministero di Cappellano della stessa. Con lui ebbi la gioia di condividere l'assidua frequentazione e l'amicizia con il Card. Carlo Maria Martini e di attingere nutrimento concreto per la nostra fede vissuta nel quotidiano, grazie alla cultura, alla visione biblica e lungimirante che caratterizzava la pastorale universitaria dell'Arcivescovo.

Il prof. Mantegazza si distinse per la lunga consuetudine di amore familiare vissuto anche nel momento drammatico della morte dei due figli, in profonda comunione di fede nel Signore Risorto, condivisa nella discrezione più intima con la moglie Andreina. Esperienza di un dono che, pur con le lacrime agli occhi, gli fece sgorgare dal cuore un grazie riconoscente e pieno di abbandono per la speranza che la fede nell'incontro con Cristo, anche secondo la tonalità del carisma di Don Giussani, mette nell'animo umano. Ogni mattina Egli passava per la Cappella, leggeva il Vangelo, mi dava un cordiale saluto mentre ci 
aggiornavamo reciprocamente su quanto atteneva la vita della Comunità studentesca e professorale.

Personalmente, egli è stato per me un 'padre' che mi ha accompagnato discretamente ma con intelligenza e generosa disponibilità nel difficile ministero della Cappellanìa Universitaria negli anni dall'86 al '95. Ho sempre con me la lettera (solo da Lui appena firmata a causa della mano malferma) con cui partecipò alla mia ordinazione episcopale nel 2007.

2. Insieme a Lui, ebbi l'onore di poter organizzare Convegni facendo tesoro delle lettere pastorali con le quali il Card. Martini, pastore attento e provvido, animava la vita spirituale culturale sociale dell'immensa nostra diocesi di Milano.

Coinvolgendo docenti e studenti, si realizzarono le Scuole per l'ascolto della Parola di Dio, fucine di studio e di meditazione che formarono decine di giovani (sia quelli provenienti da movimenti studenteschi, che quelli più lontani) alla lettura meditativa e performativa della Parola biblica. Anche da questo impegno, scaturì l'esigenza di costituire un Comitato etico all'interno degli organismi dell'Università Statale con lo scopo di indagare le problematiche etiche e le valenze giuridico-morali proprie ai vari ambiti afferenti alla medicina, alle scienze sperimentali, alle discipline umanistiche, alle leggi e alla vita sociale.

Venivano posti sul tappeto i più scottanti problemi della nostra vita contemporanea ma il metodo di un dialogo serio e costruttivo permetteva a tutti gli orientamenti morali, teoretici, scientifici e culturali di raggiungere proposte condivisibili, perché ispirate al rigore della verità e al rispetto delle coscienze. Una discreta problematicità faceva sorgere la possibilità che il Vero possa crescere sempre più e diventare un'opzione per una vita più bella e umana. Anche l'esperienza cristiana rendeva più luminose certe domande!

3. L'educazione integrale della persona caratterizzò sempre l'interesse e lo sforzo del Prof. Mantegazza. Accanto al generico compito dell'istruire, egli si prefissò quello più complesso dell'educare, a partire dalla costituzione di una vera comunità accademica tra docenti e discenti. L'intento che lo animò fu quello di non limitarsi a formare degli "eruditi", ma dei "colti" capaci cioè non solo di trattenere concetti tecniconozionistici, ma di formulare un pensiero critico costruttivo e di elaborare le competenze acquisite investendole ed adattandole alle mutevoli circostanze della vita personale e professionale. 
Il Rettore Mantegazza visse il suo impegno di educatore rispondendo quasi ad una chiamata interiore, rincorrendo un'ideale che senza esitazione vorrei definire missionario per apertura e lungimiranza. Egli riteneva essenziale umanizzare l'insegnamento universitario non necessariamente attraverso l'incontro personale con i singoli studenti (non sempre possibile a causa dei molti impegni e della massa di studenti della Statale di allora, finché non arrivò la Bicocca!), ma proprio per il tramite di quanto dalla cattedra il docente ha la possibilità di trasmettere a coloro che lo frequentano: l'esempio, la passione per lo studio e per la ricerca, la qualificazione rigorosa dell'apprendimento, la rettitudine morale nell'impegno quotidiano, la dimensione etica del sapere e dell'agire. Tutto questo in un clima di rispettosa libertà di pensiero e di opinioni al quale la stessa Università ha il compito di educare, liberando docenti, studenti e organismi accademici da pregiudiziali e condizionamenti ideologici, politici, confessionali o dottrinali.

4. La vicinanza del Prof. Mantegazza lasciò in me il tratto indelebile di un uomo mite il quale, ispirato da profonde scelte di buona vita cristiana, sapeva mettersi di fronte ai giovani universitari secondo la loro misura; e questo faceva aprire il cuore! Questo stesso atteggiamento era praticato anche nei confronti dei professori, soprattutto quando contrapposizioni, concorsi, problemi di governo e di bilancio rendevano fragili i rapporti all'interno della comunità universitaria.

5. "Beati i miti, perché avranno in eredità la terra" (Mt 5,5). Per Lui l'Università doveva educare all'universalità del sapere e al piacere di conoscere. Aborriva la distinzione tra materie umanistiche e scientifiche ritenendola impoverente e banale, mentre sosteneva con forza e convinzione l'integralità e la complementarietà delle diverse vie del sapere, tutte in egual misura necessarie alla maturazione complessiva della persona (Cfr. Francesco Giovanni Brugnaro, Educare all'università, P.M. 1991, pp. 23-28).

Per tale ragione, citava con piacere la prof.ssa Maria Corti, allora docente di Lettere all'Università di Pavia, la quale aveva scritto: «È sempre dalle materie umanistiche che è favorita la maturazione della persona, che viene dato impulso alla riflessione sul destino dell'uomo, sul significato del vivere. Sono discipline che spalancano lo sguardo sul mondo, che eliminano i paraocchi. Disprezzarle è un gravissimo errore, un allontanarsi incosciente da quella felicità umana che tutti cercano nella vita. La let- 
tura di un manuale può essere utile, rispettabilissima, ma la lettura di una poesia può dare gioia. Se si toglie questo sale della cultura, c'è il rischio che nel futuro diventi troppo insipida».

Egli, nei suoi tempi, si poneva alcuni interrogativi che forse hanno anche oggi una loro attualità o perché la risposta è ancora incompiuta, o perché la modalità abbisogna d'un inveramento completamente nuovo e più coraggioso. L'eco di queste domande giunge fino a noi:

- È cresciuto il senso del dovere nei docenti e nei discenti?

- La comunità tra studenti universitari e docenti offre adeguata soddisfazione alle giuste aspirazioni di crescita e di formazione umana, culturale ed umana di entrambe le componenti?

- La nostra Università sa essere una comunità nella quale la globalità del pensiero, il rigore del metodo e l'universalità del sapere si compiono?

- Essa è davvero sede dove i giovani imparano ad elaborare ciò che hanno studiato e sanno maturare una personalità che si manifesta attraverso un'onesta e competente professionalità?

- Stiamo lavorando davvero per un nuovo umanesimo della medicina?

... secondo l'opinion mia, a chi vuol una cosa ritrovare, bisogna adoperar la fantasia (Galileo Galilei) 